\title{
Dermoscopy of nodular skin metastases from the gastrointestinal primary cancer
}

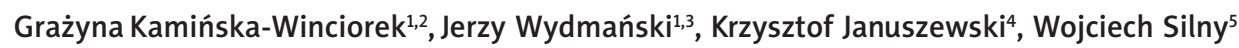

\author{
${ }^{1}$ The Center for Cancer Prevention and Treatment, Katowice, Poland \\ Head of the Center: Beata Wydmańska \\ ${ }^{2}$ Department of Bone Marrow Transplantation and Oncohematology, The Maria Sklodowska-Curie Memorial Cancer Center \\ and Institute of Oncology Gliwice Branch, Gliwice, Poland \\ Head of the Department: Prof. Sebastian Giebel MD, PhD \\ ${ }^{3}$ Department of Conventional and Intraoperative Radiotherapy, Maria Skłodowska-Curie Memorial Cancer Center and Institute \\ of Oncology Gliwice Branch, Gliwice, Poland \\ Head of the Department: Prof. Leszek Miszczyk MD, PhD \\ ${ }^{4}$ Department of Pathology, Silesian Medical Center, Silesian Medical University, Katowice, Poland \\ Head of the Department: Krzysztof Januszewski MD, PhD \\ "Great Poland Center of Allergology and Aesthetic Dermatology "Art Clinic", Poznan, Poland \\ Head of the Center: Pawet Silny MD, PhD
}

Postep Derm Alergol 2015; XXXII (4): 312-316

DOI: 10.5114/pdia.2015.48043

Dermoscopy is a useful, sufficient and widely used tool for examining pigmented and non-pigmented lesions $[1,2]$. It may be helpful as a diagnostic method in the investigation of non-characteristic skin nodules, occurring as solitary, pale or pinkish lesions, especially those of unknown origin or in the event of dealing with an unclear patient history. In the literature, there are only several case studies which have been published on the subject of dermoscopic images of metastatic nodules from internal malignancies [3-5]. Skin metastases occur in $0.6-10 \%$ of all patients with cancer and represent $2 \%$ of all skin tumors [6]. Among gastrointestinal tract malignancies, large intestine cancer among women and cancer of the esophagus, pancreas and stomach cancer among men can often metastasize to the skin [6]. Skin metastases from visceral malignancies are important for dermatologists and oncologists because of their variable clinical appearance and presentation as well as frequent delay or even failure to diagnose them correctly [6]. Metastatic skin nodules occur as non-painful, single or multiple, hard or flexible, tiny, pinkish, small nodules, without any specific clinical diagnostic criteria [4]. Clinical diagnosis in such cases is not clear. From a histopathologic point of view, there are 4 main morphological patterns of cutaneous metastases involving the dermis, namely a nodular, diffuse, infiltrative and intravascular pattern [6]. In dermoscopic evaluation of non-pigmented skin nodules, vascular structures have often played a key diagnostic role, also in the diagnostic management of metastatic skin tumors $[7,8]$.
The authors report two clinically unspecific cases of dermoscopy, with the presence of a metastatic skin cancer derived from the gastrointestinal tract.

Clinical examination with dermoscopy was performed in both of the cases presented below. Dermoscopic images from each lesion were obtained using a lens (Dermoscope Delta 20; Heine, Herrsching, Germany), mounted on a Nikon D 700 camera.

Totally excised skin nodules were examined histopathologically with the use of a routine histopathological technique in hematoxylin-eosin staining $(H+E)$. Histopathological specimens were sequentially examined field-by-field along the whole section length of the slide. This technique enables us to compare histopathological and dermoscopic pictures.

This is the case of a 60-year-old woman diagnosed with stage IV $\mathrm{PT}_{3} \mathrm{~N}_{1} \mathrm{M}_{1}$ sigmoid colon cancer. A tumor resection was performed and palliative chemotherapy undertaken between April and November 2010. Complete regression of metastases to the lung was thus achieved. Nine months later, she was found to have a recurrence of the cancer in her lung and ovary. After 6 courses of second-line chemotherapy, a small reddish nodule in the periumbilical area was observed. Clinical examination revealed a solitary, small, palpable, firm, painful nodule of $1.5 \mathrm{~cm}$ in diameter (Figure 1). Dermoscopic examination of the skin nodule manifested the presence of polymorphous vessels. Irregular, linear vessels were also observed in the area (linear straight and linear helical vessels)

Address for correspondence: Grażyna Kaminska-Winciorek MD, PhD, The Center for Cancer Prevention and Treatment, 16 Fliegera St, 40-060 Katowice, Poland, phone: +48 698626 208, fax: +48 322068 113, e-mail: dermatolog.pl@gmail.com Received: 17.02.2014, accepted: 17.07.2014. 


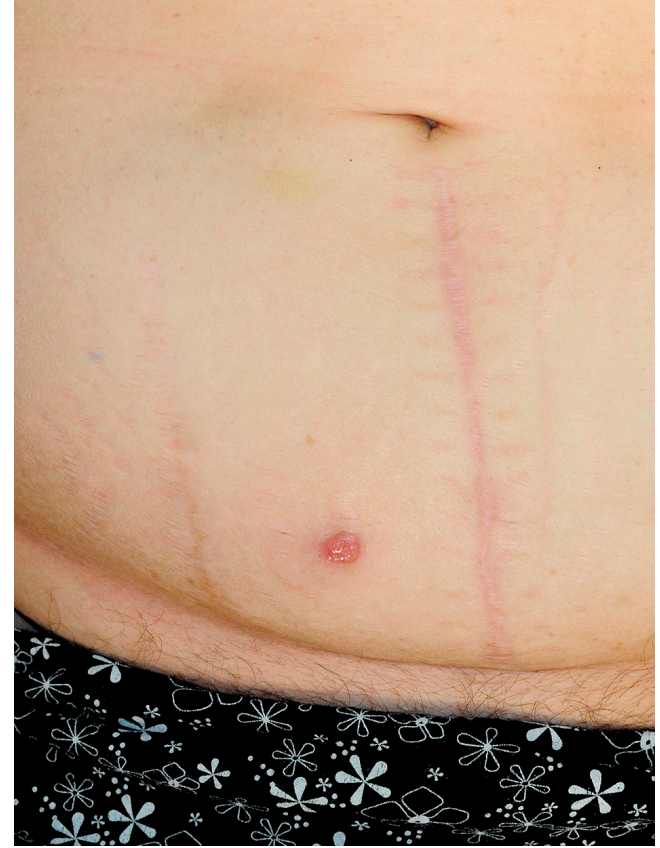

Figure 1. A case of a macroscopic skin metastatic nodule from sigmoid colon cancer. This case shows a solitary, small, palpable, firm, slightly painful nodule of $1.5 \mathrm{~cm}$ in diameter in the periumbilical area originating from sigmoid colon cancer in stage IV pT3N1M1 in a 60-year-old female patient

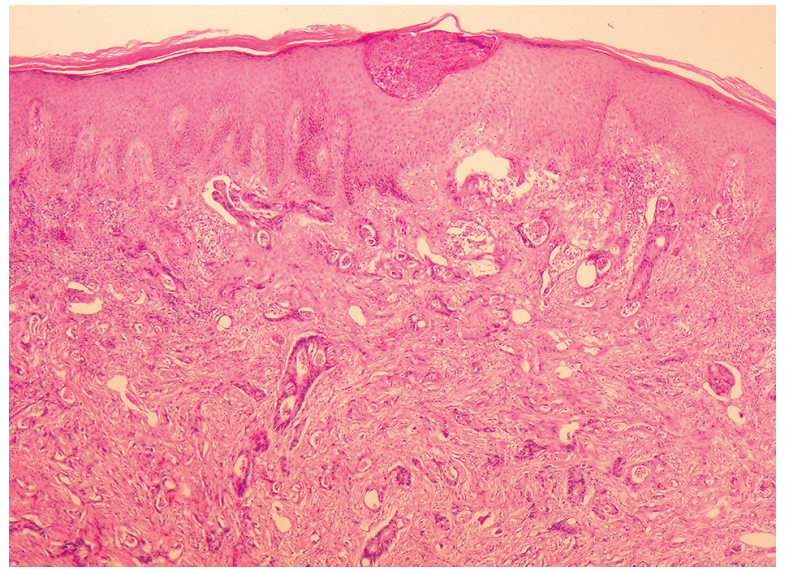

Figure 3. Magnification 100x, H + E. In the superficial part of the epidermis, a slight scaling with superficial subcorneal pustules with granulocytic debris was noted. A slight acanthotic proliferation of the epithelium was also found. In the superficial part of the dermis vessels' proliferation was also observed. In the lumina of the curved and dilated, spirally elongated vessels, well-visible multiple, metastatic carcinomatous emboli were found in the central part of the lesion, where the neovascularization was the most pronounced

mimicking stellate telangiectasias as well as some dotted (red dots) and distributed peripherally (Figure 2). The vascular arrangement was irregular and chaotic. In all parts of the lesion, the background was whitish,

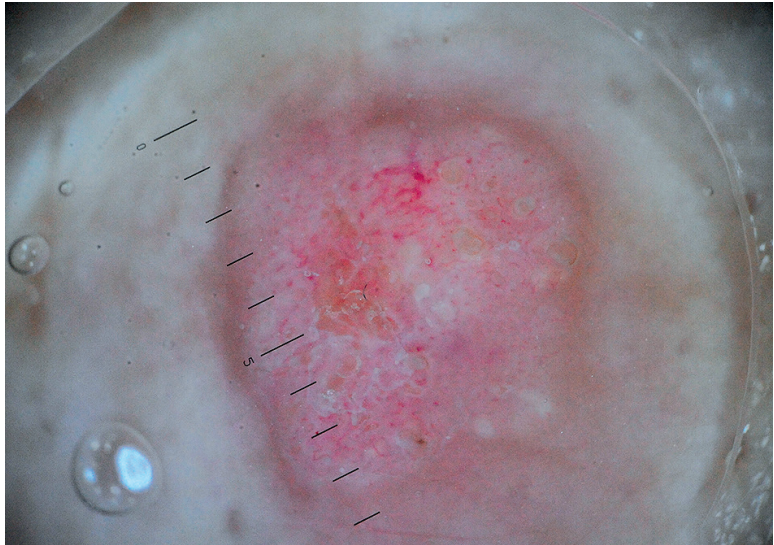

Figure 2. A case of dermoscopy of a skin metastatic nodule from sigmoid colon cancer. This figure presents multiple linear, irregular vessels located centrally and peripherally mimicking stellate telangiectasias as well as dotted vessels, mainly distributed peripherally. The vascular arrangement is irregular. The background is whitish, confluent and homogenous. Partially small, roundish, yellowish dots are observed mimicking the hyperkeratotic plugs which are surrounded by a white halo

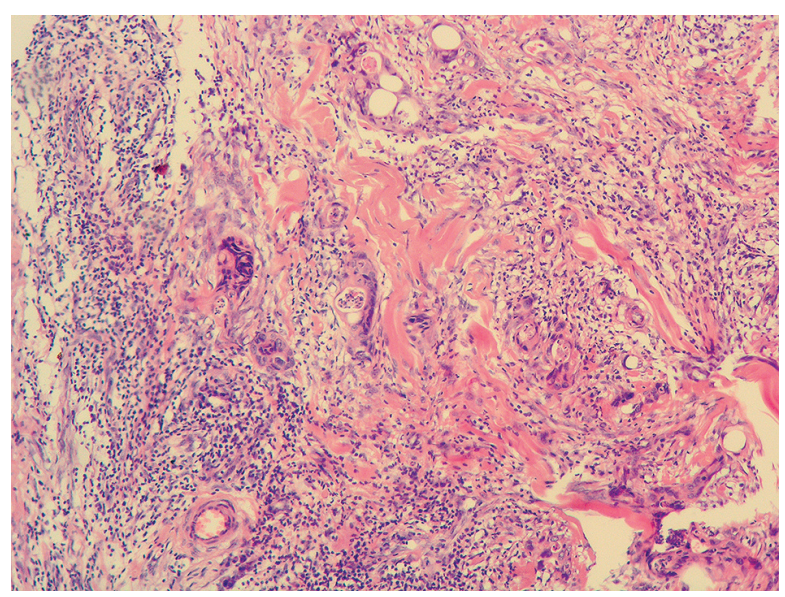

Figure 4. Magnification $100 \times, H+E$. In the epidermis, proofis observed of the presence of a dense, inflammatory infiltration, thickened and hyalinizated bundles of collagen and adenocarcinomatous tubules

confluent and homogenous. Partially small, roundish, yellowish dots were noted mimicking the yellowish hyperkeratotic plugs that were surrounded by a white halo. Because of the exacerbation of the pain caused by the skin lesion, the patient underwent a full surgical excision of the nodule. Histopathological examination of the skin tumor confirmed metastases from sigmoid colon cancer. A slight scaling with superficial subcorneal pustules with granulocytic debris were noted in the superficial part of the epidermis. A slight acanthotic proliferation of the epithelium was found. Proliferation has been observed in the superficial part of the dermis vessels. Also, well- 


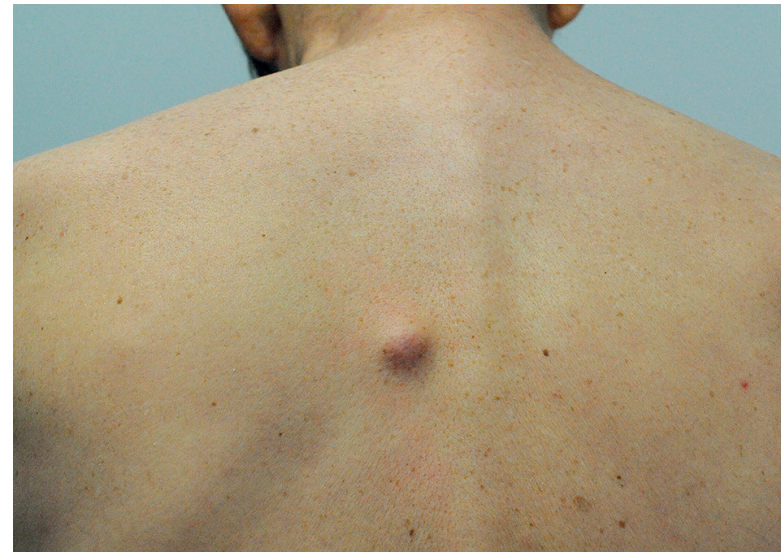

Figure 5. An example of a macroscopic picture of a metastatic nodule originating from adenocarcinoma of the pancreas. The figure presents a metastatic nodule as nonpainful, brownish-pinkish, slightly firm, non-shifting within the skin, sized $2.0 \mathrm{~cm}$ in diameter, found on the back of a 61-year-old man

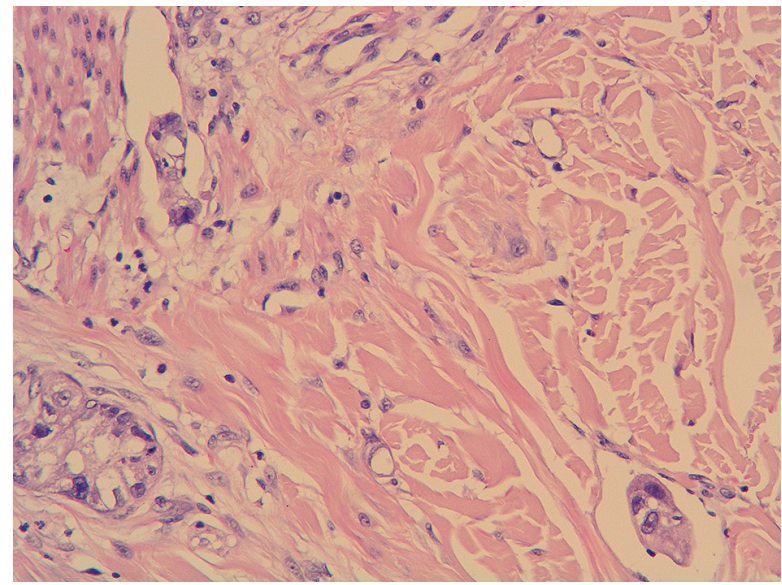

Figure 7. Magnification 200x, H + E. In the superficial part of the dermis, proliferating, curved, slightly dilated and elongated vessels with metastatic, carcinomatous emboli have been noted. Interstitial fibrosis with hyalinization has also been found

visible multiple, metastatic carcinomatous emboli were found in the lumina of the curved and dilated, spirally elongated vessels. In the central part of the lesion, the neovascularization was the most pronounced (Figure 3). In the epidermis, proof was found for the presence of a dense, inflammatory infiltration, thickened and hyalinized bundles of collagen and adenocarcinomatous tubules (Figure 4).

The patient was directed to a third line of chemotherapy.

A 61-year-old male patient was diagnosed with a tumor of the pancreas, abdominal lymphadenopathy and multiple liver tumors. The patient was not qualified for a biopsy of the tumors and was sent to an oncologist.

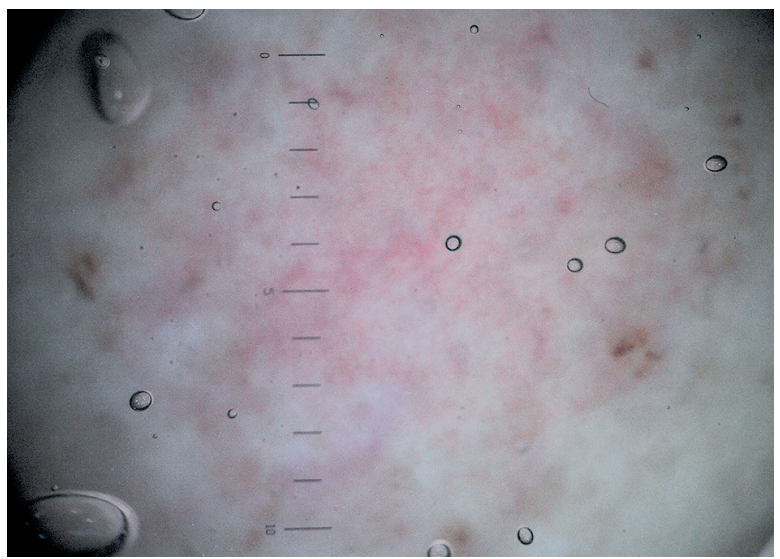

Figure 6. A case of dermoscopy of a skin metastatic nodule from pancreatic cancer. This case presents a dermoscopy of the metastatic skin nodule, which reveals the presence of polymorphous vessels, whose picture remains unclearmainly irregularly distributed linear straight and irregular, linear serpentine vessels. After the application of slight pressure, the vessels vanished rapidly. The background of the nodule is whitish and structureless, with several brownish-greyish, roundish areas, irregular in shape, similar to peripherally distributed small dots

Under clinical examination, further four skin tumors were found on his back. The nodules were non-painful, brownish-pinkish, slightly firm, non-shifting within the upper layer of the skin, sized up to $2.0 \mathrm{~cm}$ in diameter (Figure 5). A dermoscopy of the metastatic skin nodules showed the presence of a grouping of polymorphous, vaguely visible vessels mainly irregularly distributed, linear straight and linear serpentine in their form. After the application of slight pressure, the vessels rapidly vanished (Figure 6). The background of the nodules was whitish and structure less with several (4) brownish-gray, small roundish dots, irregular in shape and distributed peripherally. The largest tumor was excised and histopathologic examination proved metastatic adenocarcinoma originating from the pancreas. Based on dermoscopy conducted for histopathologic purposes, the final diagnosis of the type of pancreatic malignancy was performed and the patient was qualified for chemotherapy. In a histopathological examination in the superficial part of the dermis, proliferating, curved, slightly dilated and elongated vessels with metastatic, carcinomatous emboli were observed. Interstitial fibrosis with hyalinization has also been indicated (Figure 7). No secondary melanocytic colonization was found in this case.

The results of our case studies described two different dermoscopic images of metastatic skin nodules, which originated from cancer of the gastrointestinal tract. Dermoscopy revealed the presence of three predominant types of vascular structures. The first of these was an irregular, linear morphology. The second one was a dotted morphology (red dots), observed as multiple tiny 
red points, peripherally positioned next to each other. The third one took the form of both a linear straight and a linear serpentine. There is a relation between dermoscopic vascular structures and their histopathological features, seen as neovascularization with numerous curved, dilated vessels with inner metastatic emboli in both of the presented cases. All these vascular structures were distributed along with whitish, confluent, structureless depigmentation. Histopathologically, it could correspond to fibrosis of the dermis with the hyalinization of bundles of collagen. In our experience, previously described cases of metastatic breast cancer were also characterized by the occurrence of polymorphous vessels located on a whitish background [4]. According to Zalaudek et al. [7, 8], the presence of linear irregular and dotted vessels, located centrally or irregularly, is commonly present in the case of thin or intermediate a melanocytic or hypomelanocytic melanoma. Also, the occurrence of regular, dotted vessels with positive additional dermoscopic criteria such as reticular depigmentation, chrysalis structures and depigmentation - all these may strongly suggest a melanocytic or hypomelanocytic melanoma or the Spitz nevus [7]. In differential diagnosis, mycosis fungoides lesions exhibited a characteristic dermoscopic pattern consisting of fine, short linear vessels, and combined vascular structures composed of dotted and curved linear vessels occurring in patchy, orange-yellowish areas [9].

Arborizing vessels are a specific finding of the nodular cystic or the scleroderma-like basal cell carcinoma, or fine "microarborizing" vessels, with shiny red-white structureless areas, which are typical of the superficially spreading type of basal cell carcinoma [7, 8]. The feature which led to the exclusion of basal cell carcinoma (BCC) diagnosis was the absence of erosions [7, 8]. The pigmented brownish-grayish dots and globules resembling residuals of hyperpigmentation located peripherally, which were presented in our case study, were not characteristic of melanoma or pigmented variants of BCC $[10,11]$. They were not similar to the previously described multiple blue or gray globules, leaf- like areas and spoke-wheel areas most commonly found in basal cell carcinoma [10, 11]. Dermoscopy of fibroepithelioma of Pinkus as a rare variant of $\mathrm{BCC}$ is also characterized by the occurrence of polymorphous vessels mainly consisting of fine, focused, short arborizing and dotted vessels, the latter mainly located peripherally, with visible short, white streaks [12].

Atypical, polymorphous vascular patterns with irregular linear-vessels, milky-red areas and globules can all be suggestive of a malignant process, also in the case of the Merkel cell carcinoma [13].

Perhaps it is possible that in the cases presented herein, the shape and type of the vessels depend on the stage of the nodule. Similarly to melanoma in its recent stages, dotted vessels are predominant [11]. Vessels characteristic of advanced tumors are wider, coarse, more irregular and variable [11]. To date, only dermoscopic features of metastatic melanoma have ever been widely described [14-17]. A clue for cutaneous melanoma metastases in differential diagnosis of bluish, purple or red nodules with recent onset may be found in the form of the presence of stellate telangiectasias surrounding the lesion [17]. In this case, linear, irregular prominent vessels or stellate telangiectasias at the periphery surrounded by a structureless metastatic lesion are typical [17]. The predominant dermoscopic feature of a melanotic cutaneous metastases of melanoma was the presence of vascular structures, including serpentine, glomerular, irregular hairpin and corkscrew-like vessels [14]. Recently described metastatic melanoma patterns by Costa et al. [18] also took into account pinkish variants on dermoscopy [18]. The vascular variant is characterized by three or more atypical vessels (dotted, linear irregular, corkscrew, irregular/polymorphous) with light brown pigmentation without globules [18]. In unspecific patterns of metastatic melanoma, the presence of 3 or more nonspecific vessels, homogeneous brown pattern were present, there were no other patterns to be noted [18].

Few publications exist concerning the dermoscopy of metastatic tumors, especially the metastatic solid tumor. Previously de Giorgi et al. [3] described a dermoscopic pattern of cutaneous metastatic thyroid cancer which revealed the presence of atypical and polymorphous vascular structures within the pink, solitary, erythematous lesion. Also Wyatt et al. [5], Kaminska-Winciorek and Wydmanski [4] have presented dermoscopic images of skin metastatic nodules derived from a mammary carcinoma. The combination of two vascular types in metastatic skin cancer originating from the gastrointestinal tract indicated a polymorphous type of vessels. Solitary nodular metastases originating from cancers of the pancreas and large intestine should be taken into account in the differential diagnosis of new or persistent skincolored or pink papules or nodules in the case of patients with a previous history of internal malignancy, similarly to metastases in melanoma [14].

Polymorphous vessels on dermoscopy in metastatic nodules derived from gastrointestinal malignancies were first described in 2013. Mun et al. [19] reported dermoscopic images of the umbilical Sister Mary Joseph nodule proved histopathologically to be a metastatic adenocarcinoma. Similarly, this lesion is also present in our cases, showing up on dermoscopy asa polymorphous vascular pattern [19]. Also Ito et al. [20], on dermoscopy of peristomal metastasis from the adenocarcinoma of the colon, discovered a series of randomly arranged, polymorphous vessels and irregular white-to-pink structures [20]. The presence of polymorphous vessels, similarly to our reported cases, is most probably the most commonly occurring dermoscopic feature of metastatic nodules derived from cancer of the gastrointestinal tract.

One of the limitations of our study is that the finding of dotted vessels or coarse vessels in itself tends to be 
lightly non-specific. Also, the "strawberry pattern" described earlier, characterized by a background erythema and red pseudo-network consisting of unfocused, large vessels located between the hair follicles, associated with prominent follicular openings surrounded by a white halo may occur in non-pigmented actinic keratosis [21, 22]. In a histopathological assessment, no features of secondary melanocytic colonization of non-melanocytic tumors, previously described by Wyatt et al. [5] were to be found. Dermoscopy at this stage does not offer much help without any histopathological confirmation, however, in the aspect of unusual clinical presentation of solitary, pinkish nodules, revealing polymorphous vessels leads to a more thorough diagnostic process and increases the index of suspicions of malignancies.

Our case study proved dermoscopy as an additional diagnostic technique in the differential diagnosis of pink, solitary nodules in its metastatic morphology, likely to be recognized not only as melanoma in its early stages [23] but also as other rare dermatological or mucosal disorders [24, 25].

Knowledge of the occurrence of metastatic skin tumors from internal malignancies is essential. Dermoscopy illustrates abnormal polymorphous vascular structures occurring in atypical, non-specific, solitary metastatic nodules. Histopathological confirmation is mandatory for all lesions displaying polymorphous vessels. Furthermore, a study of cutaneous metastases should also be included in the differential diagnosis. Due to the fact that this is one of the first reports of its kind, further research and observation are still needed.

\section{Conflict of interest}

The authors declare no conflict of interest.

\section{References}

1. Kaminska-Winciorek G, Spiewak R. Tips and tricks in the dermoscopy of pigmented lesions. BMC Dermatol 2012; 12: 14.

2. Kaminska-Winciorek G, Spiewak R. Basic dermoscopy of melanocytic lesions for beginners. Postepy Hig Med Dosw (Online) 2011; 65: 501-8.

3. de Giorgi V, Alfaioli B, Massi D, et al. Solitary cutaneous metastasis as the first sign of relapse of thyroid carcinoma: a clinical, dermoscopic-pathologic case study. Dermatol Surg 2009; 35: 523-6.

4. Kaminska-Winciorek G, Wydmanski J. Dermoscopy of skin metastases from the breast cancer and the orange pee type ("peaud orange"): a report of two cases. Int J Dermatol 2015; 54: 343-6.

5. Wyatt AJ, Agero AL, Delgado R, et al. Cutaneous metastatic breast carcinoma with melanocyte colonization: a clinical and dermoscopic mimic of malignant melanoma. Dermatol Surg 2006; 32: 949-54.

6. Alcaraz I, Cerroni L, Rütten A, et al. Cutaneous metastases from internal malignancies: a clinicopathologic and immunohistochemical review. Am I Dermatopathol 2012; 34: 347-93.
7. Zalaudek I, Kreusch J, Giacomel J, et al. How to diagnose nonpigmented skin tumors: a review of vascular structures seen with dermoscopy: part I. Melanocytic skin tumors. J Am Acad Dermatol 2010; 63: 361-74.

8. Zalaudek I, Kreusch J, Giacomel J, et al. How to diagnose nonpigmented skin tumors: a review of vascular structures seen with dermoscopy: part II. Nonmelanocytic skin tumors. J Am Acad Dermatol 2010; 63: 377-86.

9. Lallas A, Apalla Z, Lefaki I, et al. Dermoscopy of early stage mycosis fungoides. J Eur Acad Dermatol Venereol 2013; 27: 617-21.

10. Altamura D, Menzies SW, Argenziano G, et al. Dermatoscopy of basal cell carcinoma: morphologic variability of global and local features and accuracy of diagnosis. J Am Acad Dermatol 2010; 62: 67-75.

11. Argenziano G, Albertini G, Zalaudek I. Commentary: improved detection of nonpigmented skin tumors. Dermatol Surg 2012; 38: 1445-7.

12. Reggiani C, Zalaudek I, Piana S, et al. Fibroepithelioma of pinkus: case reports and review of the literature. Dermatology 2013; 226: 207-11.

13. Harting MS, Ludgate MW, Fullen DR, et al. Dermatoscopic vascular patterns in cutaneous Merkel cell carcinoma. J Am Acad Dermatol 2012; 66: 923-7.

14. Jaimes N, Halpern JA, Puig S, et al. Dermoscopy: an aid to the detection of a melanotic cutaneous melanoma metastases. Dermatol Surg 2012; 38: 1437-44.

15. Watanabe T, Higaki H, Yamada N, et al. Dermoscopic and histopathological findings of epidermotropic metastatic malignant melanoma. Eur J Dermatol 2011; 21: 811-3.

16. Minagawa A, Koga H, Sakaizawa K, et al. Dermoscopic and histopathological findings of polymorphous vessels in a melanotic cutaneous metastasis of pigmented cutaneous melanoma. Br J Dermatol 2009; 160: 1134-6.

17. Julian Y, Argenziano G, Moscarella E, et al. Peripheral stellate telangiectasias: a clinical-dermoscopic clue for diagnosing cutaneous melanoma metastases. I Dermatol Case Rep 2012; 6: 102-4.

18. Costa J, Ortiz-Ibańez K, Salerni G, et al. Dermoscopic patterns of melanoma metastases: interobserver consistency and accuracy for metastasis recognition. Br J Dermatol 2013; 169: 91-9.

19. Mun JH, Kim JM, Ko HC, et al. Dermoscopy of a Sister Mary Joseph nodule. J Am Acad Dermatol 2013; 68: e190-2.

20. Ito T, Yoshida Y, Yamada N, et al. Dermoscopy of peristomal polyps and metastasis of colon cancer. Acta Derm Venereol 2014; 94: 96-7.

21. Zalaudek I, Giacomel J, Argenziano G, et al. Dermoscopy of facial nonpigmented actinic keratosis. Br J Dermatol 2006; 155: 951-6.

22. Peris K, Micantonio T, Piccolo D, Fargnoli MC. Dermoscopic features of actinic keratosis. J Dtsch Dermatol Ges 2007; 5: 970-6.

23. Kamińska-Winciorek G, Właszczuk P, Wydmański J. "Mistletoe sign": probably a new dermoscopic descriptor for melanoma in situ and melanocytic junctional nevus in the inflammatory stage. Postep Derm Alergol 2013; 30: 316-9.

24. Drogoszewska B, Chomik P, Michcik A, Polcyn A. A standard picture of healthy oral mucosae by direct oral microscopy. Postep Derm Alergol 2013; 30: 159-64.

25. Kamińska-Winciorek G, Spiewak R. Dermoscopy on nevus comedonicus: a case report and review of the literature. Postep Derm Alergol 2013; 30: 252-4. 